
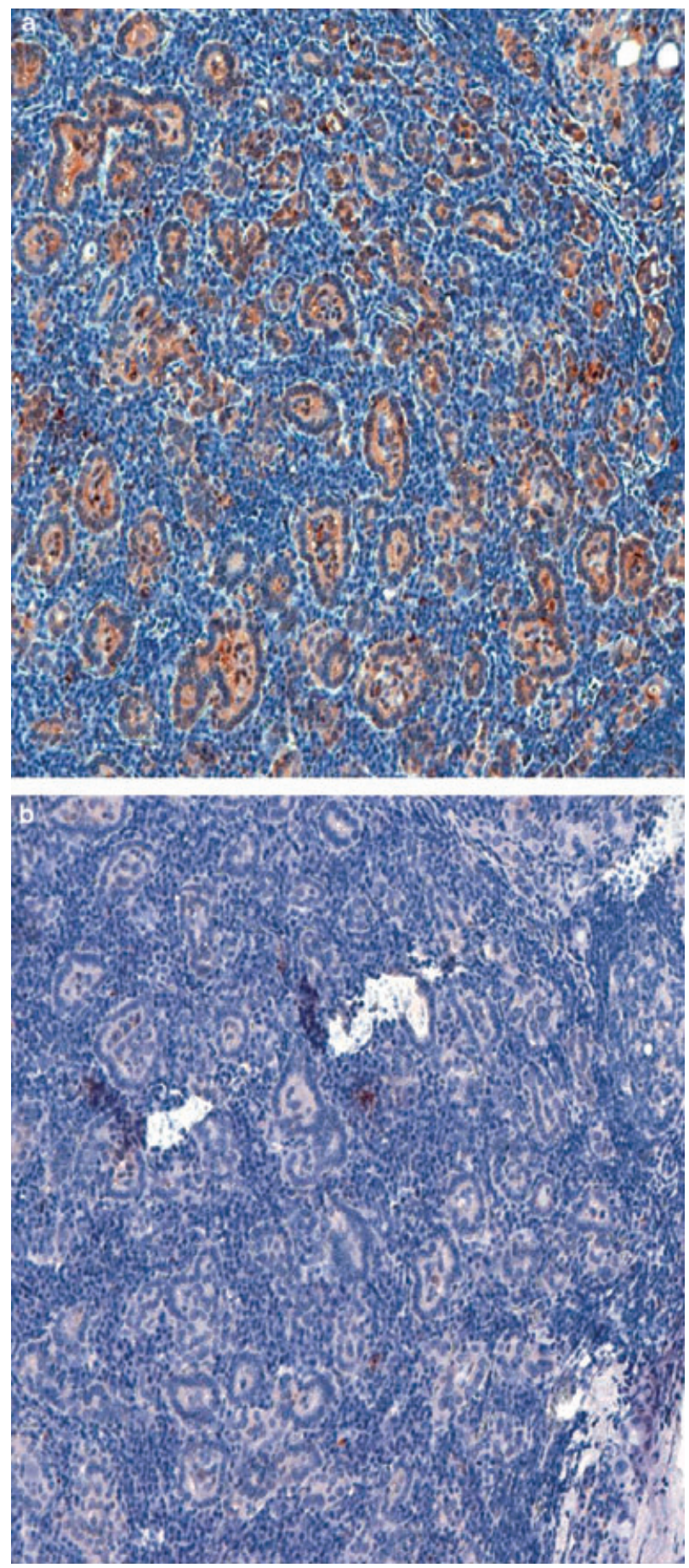

Figure 1. a, Galectin-3 immunostaining of Hashimoto's thyroiditis by using a purified $\mathrm{mAb}$ to galectin- 3 and the avidin-biotin complex method (indirect immunoperoxidase). Similar staining was obtained using an unrelated $\mathrm{mAb}$ as control (data not shown). $\mathbf{b}$, The immunoreactivity of the same mAb to galectin-3 when used in a biotin-free indirect immunoperoxidase with a secondary antibody, horseradish peroxidase conjugated. analysis confirmed both the specificity of the antiserum and expression of galectin-3 in multinodular goitres, follicular adenoma and carcinomas. The galectin-3 mAb they used is specific for galectin-3 (although this reagent is not purified and needs to be tested in different dilutions), but Western blotting procedures are not adequate to support the authors' conclusions. ${ }^{4}$

We agree with the authors that further studies are needed in order to confirm the value of galectin-3 immunostaining in clinical practice. For this reason, we are now engaged in a prospective multicentre study. However, reliability and reproducibility of a diagnostic test require stringent methodological procedures including optimal substrates and purified and standardized reagents. Moreover, for this specific field, biotin-free staining procedures are mandatory.

A Bartolazzi
G Bussolati

Department of Pathology, St Andrea University Hospital, Rome, and

${ }^{1}$ Department of Pathology, University of Turin, Turin, Italy

1. Mehrotra P, Okpokam A, Bouhaidar R et al. Galectin-3 does not reliably distinguish benign from malignant thyroid neoplasms. Histopathology 2004; 45; 493-500.

2. Bussolati G, Gugliotta P, Volante M, Pace M, Papotti M. Retrieved endogenous biotin: a novel marker and a potential pitfall in diagnostic immunohistochemistry. Histopathology 1997; 31; 400407.

3. Herrmann ME, LiVolsi VA, Pasha TL, Roberts SA, Wojcik EM, Baloch ZW. Immunohistochemical expression of galectin-3 in benign and malignant thyroid lesions. Arch. Pathol. Lab. Med. 2002; 126; 710-713.

4. Bartolazzi A, Papotti M, Orlandi F. Methodological considerations regarding the use of galectin-3 expression analysis in preoperative evaluation of thyroid nodules. J. Clin. Endocrinol. Metab. 2003; 88; 950-951.

\section{Colonization of epithelial pilar neoplasms by melanocytes}

DOI: $10.1111 /$ j.1365-2559.2005.02213.x

Sir: Skin adnexal tumours are thought to be rare entities, can cause diagnostic dilemmas in routine histopathological practice and are essentially recog- 
nized at low magnification (pattern recognition). ${ }^{1}$ Helpful clues include the presence of intracellular and intercellular lumina containing diastase-resistant periodic acid-Schiff (PAS)-positive material in the diagnosis of ductal tumours, foamy epithelial cells in sebaceous tumours and pilar elements in tumours with follicular differentiation. ${ }^{1,2}$ The presence of melanocytes has been reported within various tumours, sometimes resulting in the creation of entirely 'new' entities or variants in an otherwise conventional and well-recognized entity. We present a systematic study of the presence of melanocytes in cutaneous epithelial pilar neoplasms (CEPN), which was carried out to elucidate their frequency and diagnostic utility, including other adnexal tumours as control material for that purpose.

We retrieved 50 CEPN from The Royal London Hospital archives (1980-2003), 46 benign (39 trichoepitheliomas/seven trichoblastomas) and four malignant (trichilemmal carcinomas). The pilar differentiation criteria included organoid, lobular or folliculo-centric growth pattern, few or no connections with the epidermis, keratinous microcysts and 'cell balls', hyalinized stroma and catagen-like apoptotic bodies. ${ }^{1,2}$ Malignancy criteria included infiltrative margins, nuclear pleomorphism, necrosis and mitotic figures including atypical ones. ${ }^{1,2}$ Two haematoxylin and eosin (H\&E) levels were reviewed by two histopathologists (Z.A. and S.D-C.) to systematically assess growth pattern, catagen-like apoptotic bodies, dual cell population, pigment, stromal reaction (myxoid, lamellar fibrosis, hyalinization), calcification, nuclear features, mitotic figures/10 high-power fields (equivalent to $1.52 \mathrm{~mm}^{2}$ ), and immunostaining for $\mathrm{CD} 34$, S100 and HMB-45. As controls, adnexal neoplasms (eight sebaceous adenomas, two sebaceous carcinomas, seven poromas/nodular hidradenomas, three porocarcinomas/ hidradenocarcinomas) were identified according to standard criteria. ${ }^{2}$ Basal cell carcinomas $(n=10)$ were also included in the control group because they are frequently considered in the differential diagnosis of CEPN.

Peritumoral stromal induction was observed in all CEPN (myxoid in 20, 40\%; lamellar in 27, 54\%; and hyaline in 10, 20\%). All trichilemmal carcinomas revealed mixed lamellar-hyaline stromal induction, with CD34+ peritumoral spindle cells (Figure 1) 38 cases $(76 \%, 35$ benign and three malignant), representing $28.16 \%$ of the peritumoral stromal cells. All CEPN revealed a dual cell population in the tumour nests, epithelial cells revealing bland nuclei and distinct nucleolus and interstitial cells in the nests showing hyperchromatic spindle nuclei (Figure 1).
Regardless of the presence of pigment, the latter component was revealed to be positive for both $\mathrm{S} 100$ and HMB-45, representing $4.33 \%$ of the nests' cellularity. Melanin pigment was demonstrated in 17 CEPN (34\%, all benign, predominantly epithelial in seven and stromal in 10). Calcification was identified in 28 neoplasms (56\%, all benign, nine stromal and 17 epithelial in 17 cases). Catagen-like apoptotic bodies were identified in 26 CEPN (52\%, 22 benign and all four malignant) and correlated positively with the presence of epithelial pigment (71\%). Tumours revealing a multifocal growth pattern showed mitotic figures $(80 \%)$, while these were less frequently present in nonmultifocal neoplasms (49\%). The other histological variables revealed no correlations. Malignant CEPN showed infiltrative margins, a trabecular growth pattern and few keratin cysts in a focally hyalinized stroma. Marked nuclear pleomorphism, small but prominent nucleoli and focal clear cell changes were characteristic features. Numerous mitotic figures including abnormal forms were evident, as were focal areas of necrosis.

Adnexal tumours revealed no CD34+ stromal induction and melanocytes were observed only in cases with H\&E-demonstrable pigment (two pigmented poromas with an average of $1.95 \%$ of HMB- $45+$ cells in the tumour nests, Figure 2). Calcifications and catagen-like apoptotic bodies were not seen in any of the non-pilar adnexal tumours.

Both epithelial nest colonization by dendritic melanocytes with hyperchromatic nuclei and the presence of CD34+ peritumoral stromal induction are clues for the morphological recognition of CEPN. Melanocytes are rarely present within various epithelial tumours and have been reported in both cutaneous and extracutaneous neoplasms, resulting in derivation of the prefix pigmented or melanocytecontaining tumours. ${ }^{2,3}$ The systematic presence of spindle and non-atypical melanocytes in CEPN questions the separation of pigmented pilar neoplasms (e.g. melanotrichoblastoma or pilar melanocytic tumour) as independent entities. ${ }^{4,5}$ These conditions probably represent extreme forms of melanocyte colonization of CEPN and do not merit designation as 'new' entities. The presence of melanocytes in CEPN remains an intriguing question, probably related to melanocyte migration capacity, which recapitulates the physiological colonization of the follicular adnexa of skin. The most important factor is to elucidate whether the melanocytic component shows evidence of dysplasia or malignancy, using the criteria applied for these lesions in other locations. $^{6,7}$ Dysplastic and malignant changes may 

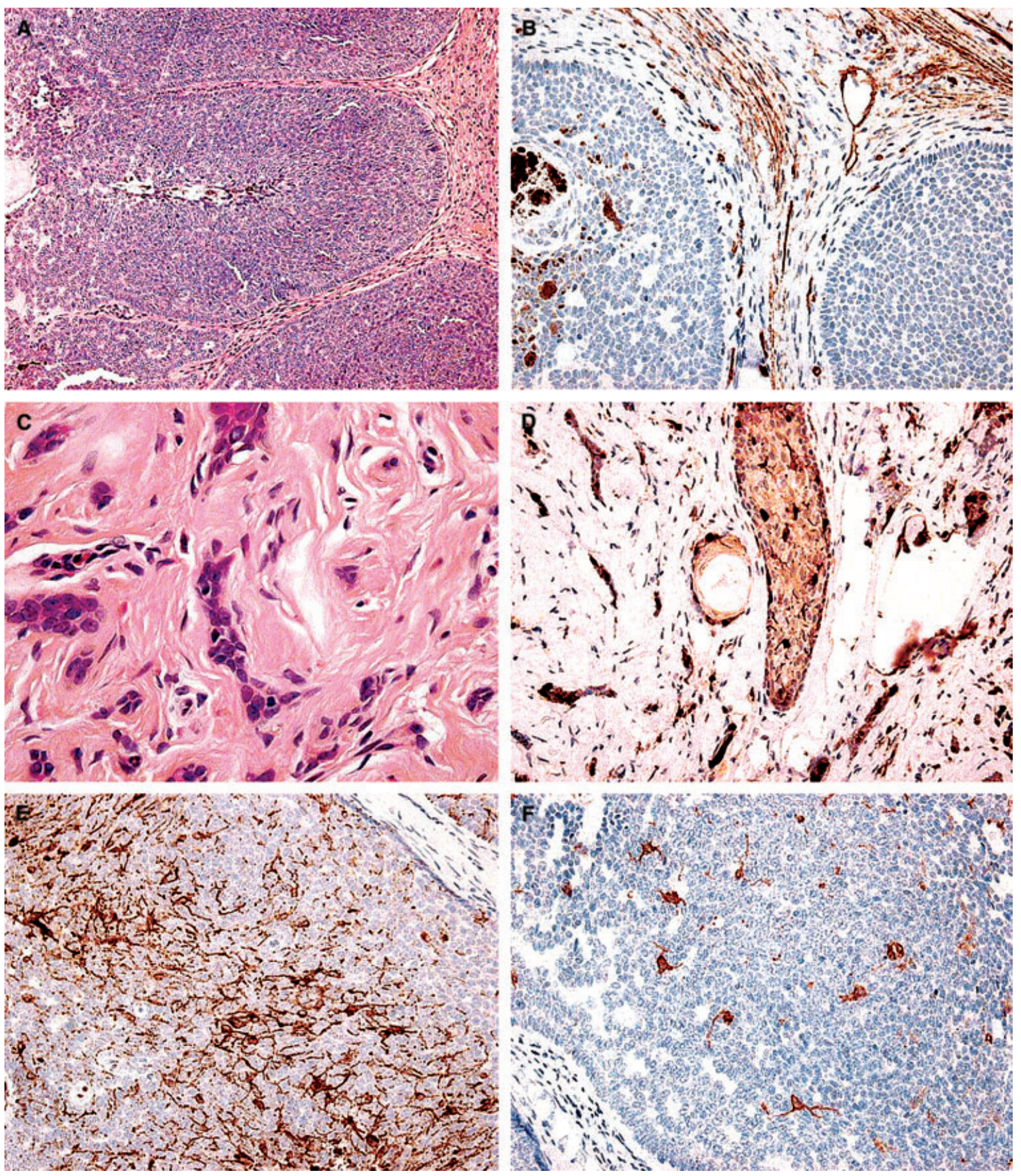

Figure 1. Melanocyte colonization in epithelial pilar neoplasms. These neoplasms frequently reveal a lobular growth pattern and stromal induction (A), along with CD34+ peritumoral stromal spindle cells (B). Tumour islands show a dual cell population: epithelioid and hyperchromatic spindle cells (C), the latter being positive for S100 (D). These spindle cells demonstrate dendritic cytoplasm and are shown to be positive for HMB-45 (E). Very occasional CD1a+ Langerhans cells are also noted in these neoplasms (F).

occur in the melanocytic component of epithelial pilar neoplasms and would explain the description biphasic neoplasm. ${ }^{4,5}$
In conclusion, melanocytes are invariably found within the epithelial nests of CEPN (both benign and malignant) and can be used along with the presence of 

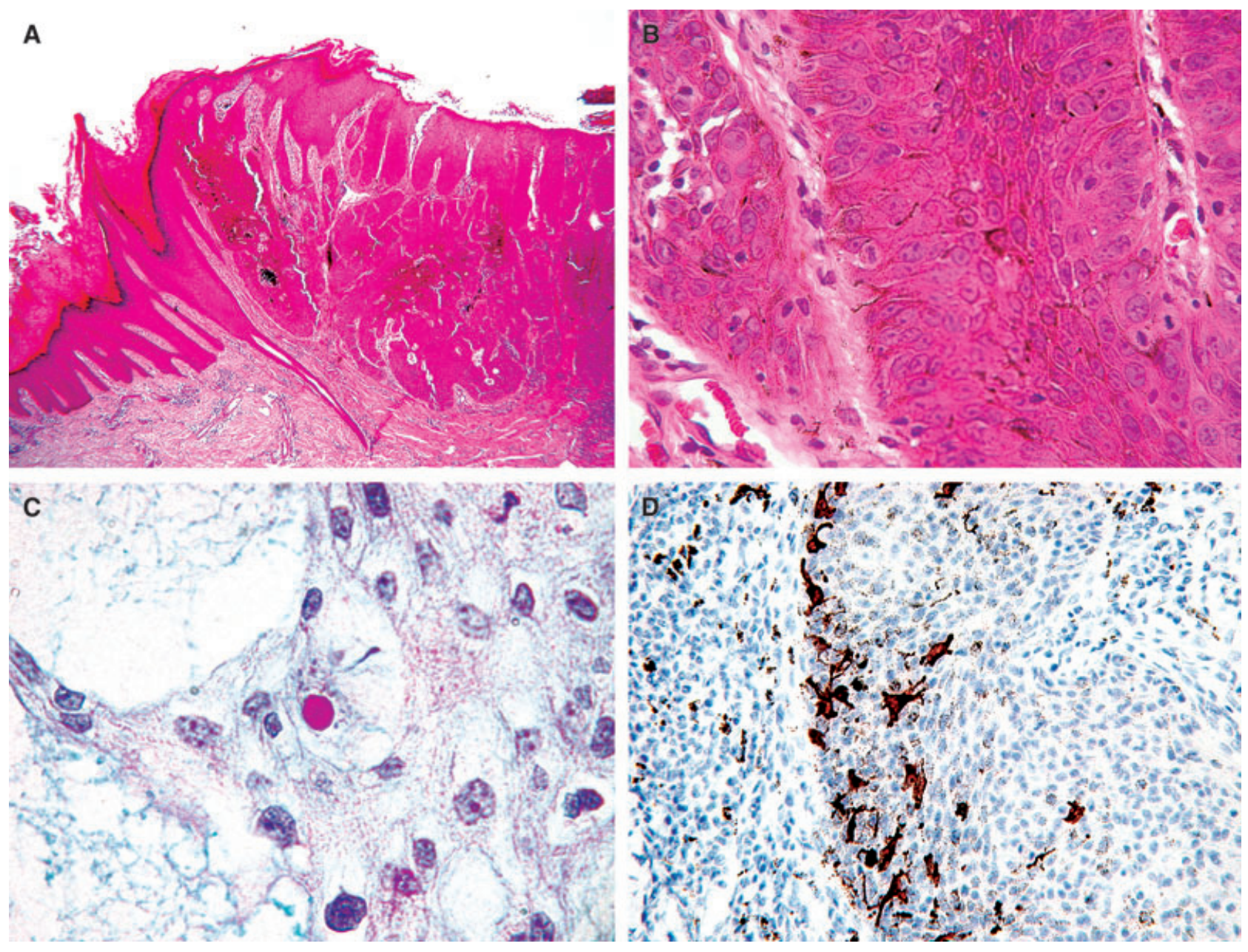

Figure 2. Melanocytes in adnexal neoplasms. Only pigmented poromas reveal melanocytes in the tumour nests. These neoplasms show a juxtaepidermal growth pattern (A), extensive melanin pigment in the epithelial tumour cells (B), and diastase-resistant periodic acid-Schiffpositive globules (C, alcian blue-DPAS). Interstitial dendritic melanocytes are also noted at the periphery of the tumour nests (D).

CD34+ stromal induction as clues for pilar differentiation, in the absence of epithelial cell melanin during routine histological examination.

\section{ACKNOWLEDGEMENT}

This work was presented in part at the XXIV International Congress of the International Academy of Pathology. Amsterdam, October 2002.

\section{Z Aly \\ L Pozo ${ }^{1}$ \\ S J Diaz-Cano}

Departments of Histopathology,

Barts and The London NHS Trust, and ${ }^{1}$ Dermatology, Homerton University Hospital,

London, UK
1. Ackerman AB, De Viragh PA, Chongchitnant N. Neoplasms with follicular differentiation (Ackerman's histologic diagnosis of neoplastic skin diseases: a method by pattern analysis), 1st edn. Philadelphia: Lea \& Febiger 1993.

2. Murphy GE, Elder DE. Nonmelanocytic tumors of the skin. In Rosai J, Sobin L, eds. Atlas of tumor pathology, 3rd series. Washington, DC: Armed Forces Institute of Pathology 1991.

3. Diaz-Cano SJ. Melanocytes in squamous cell carcinoma of the skin. Br. J. Dermatol. 2000; 142; 184.

4. Chen HM, Berney DM, Medenica M, Montag A, Krausz T. Pilar melanocytic tumor: a rare biphasic neoplasm. Lab. Invest. 2003; 83; 89A (Abstract).

5. Kanitakis J, Brutzkus A, Butnaru AC, Claudy A. Melanotrichoblastoma: immunohistochemical study of a variant of pigmented trichoblastoma. Am. J. Dermatopathol. 2002; 24; 498-501.

6. Gonzalez-Campora R, Galera-Davidson H, Vazquez-Ramirez FJ, Diaz-Cano S. Blue nevus: classical types and new related entities. A differential diagnostic review. Pathol. Res. Pract. 1994; 190; 627635. 
7. Pozo L, Diaz-Cano SJ. Malignant deep sclerosing blue naevus presenting as a subcutaneous soft tissue mass. Br. J. Dermatol. 2004; 151; 508-511.

\section{Lack of agreement on predictors for metastasizing thin melanomas}

DOI: $10.1111 /$ j.1365-2559.2005.02215.x

Sir: Most newly diagnosed melanomas are thin melanomas $(<1.0 \mathrm{~mm}$ thick) and over 95\% of these patients will still be alive at 10 years. ${ }^{1-3}$ There is a need to identify clinical and histological markers for the remaining melanomas that will metastasize and for which sentinal lymph node biopsy and adjuvant therapy may be warranted. Unfortunately, as summarized here, the literature on this subject has been contradictory.

Over the past quarter century, numerous studies have attempted to identify clinicopathological markers for progression of thin melanomas (Table 1). One prospective and several large retrospective studies have validated that a Breslow thickness (BT)

Table 1. Predictors of metastasis for thin melanoma $(<1 \mathrm{~mm})$

\begin{tabular}{lll}
\hline $\begin{array}{l}\text { Minimal or no } \\
\text { significant } \\
\text { risk factor }\end{array}$ & $\begin{array}{l}\text { Clinicopathological } \\
\text { variable }\end{array}$ & $\begin{array}{l}\text { Significant } \\
\text { risk } \\
\text { factor }\end{array}$ \\
\hline- & $\begin{array}{l}\text { Breslow thickness } \\
>0.75 \text { or } 0.8 \mathrm{~mm}\end{array}$ & $1,2,7,9,14,20$ \\
\hline- & Vertical growth phase & $3,5-9$ \\
\hline- & Mitotic rate $>(0) / \mathrm{mm}^{2}$ & $1,3-7,18$ \\
\hline $2-4,8,13,22$ & Tumour regression & $6,7,15,23-28$ \\
\hline $2-4,7,14,15$ & Ulceration & $1,5,10-13,29$ \\
\hline $1,3,14,20$ & Clark level $>$ III & $4,7,10,15-19$ \\
\hline $1,10,11,15,23,30$ & Male & $2,3,14,16,17$ \\
\hline $3,10,23,30$ & Age $>$ 45 or 60 & $1,2,14$ \\
\hline $3,10,11,15,19$ & TANS* axial or head/neck & $2,7,10,16,23$ \\
\hline 8,23 & TILst & 3,7 \\
\hline 3,15 & Acral lentiginous & 2 \\
\hline & &
\end{tabular}

*Trunk, axial, neck and shoulder.

†Tumour infliltrating lymphocytes.
$>0.75 \mathrm{~mm}$ and the presence of mitosis are negative prognostic features of thin melanomas. ${ }^{1-7}$ In a recent prospective trial, the presence of vertical growth phase was also a negative prognostic factor, ${ }^{3}$ confirming earlier powerful retrospective studies. ${ }^{5-9}$ As a sign of the confusion that has enveloped the study of thin melanomas, the two largest retrospective studies (involving 12728 and 20834 thin melanomas) contradict each other on the significance of ulceration and male sex as risk factors. ${ }^{1,2}$

Contradictory results are as striking for eight other standard clinicopathological features (Table 1). In the American Joint Committee on Cancer melanoma staging protocol, ulceration and Clark level > III are negative prognostic features. ${ }^{10}$ Several other large studies, including several with thousands of patients, support the association between ulceration and risk of recurrence. ${ }^{5,10-13}$ In contrast, other studies have shown no or minimal risk of progression in the presence of ulceration. $^{2-4,7,14,15}$ Similarly, while several large studies support invasion to Clark level IV as a risk factor, ${ }^{4,7,10,15-19}$ others, including a prospective study of 884 thin melanomas and a retrospective study of 20834 thin melanomas, do not support these findings. ${ }^{1,3,14,20}$ Similar controversies are found regarding tumour regression, male sex, older age, location of tumour, presence of tumour infiltrating lymphocytes and histologic type (Table 1).

Based on their analysis of hundreds of patients with thin melanomas, three studies have proposed criteria for stratifying risk for progession of thin melanomas (Table 2). In a retrospective study of 681 thin melanomas (127 recurrences), male sex, axial location, presence of regression and Clark level IV involvement confer a higher risk of progression. ${ }^{21}$ In a prospective trial of 884 thin melanomas (70 recurrences), the presence of mitosis, vertical growth phase (VGP) and male sex were negative prognostic factors. ${ }^{3}$ Finally, in a retrospective study of 1158 thin melanomas (177 recurrences, male sex, age $>45$ years and BT $>0.75 \mathrm{~mm}$ were negative prognostic factors. ${ }^{14}$ Once again, the lack of consistent results among the three trials is noted.

In summary, none of the standard clinicopathological risk factors has been proven to identify sensitively and specifically the small subset of thin melanomas that will metastasize. Contradictory results probably reflect the low frequency of metastasizing thin melanomas (e.g. rare disease) and the inherent differences in study design (e.g. retrospective versus prospective and case-control versus series). Perhaps novel molecular studies will prevail where routine histology and clinical features have not. 